\title{
Container productivity, daily survival rates and dispersal of Aedes aegypti mosquitoes in a high income dengue epidemic neighbourhood of Rio de Janeiro: presumed influence of differential urban structure on mosquito biology
}

\author{
Mariana Rocha David, Ricardo Lourenço-de-Oliveira, Rafael Maciel de Freitas/ ${ }^{+}$ \\ Laboratório de Transmissores de Hematozoários, Instituto Oswaldo Cruz-Fiocruz, Rio de Janeiro, RJ, Brazil
}

\begin{abstract}
Different urban structures might affect the life history parameters of Aedes aegypti and, consequently, dengue transmission. Container productivity, probability of daily survival (PDS) and dispersal rates were estimated for mosquito populations in a high income neighbourhood of Rio de Janeiro. Results were contrasted with those previously found in a suburban district, as well as those recorded in a slum. After inspecting 1,041 premises, domestic drains and discarded plastic pots were identified as the most productive containers, collectively holding up to $80 \%$ of the total pupae. In addition, three cohorts of dust-marked Ae. aegypti females were released and recaptured daily using BGS-Traps, sticky ovitraps and backpack aspirators in 50 randomly selected houses; recapture rate ranged from 5-12.2\% within cohorts. PDS was determined by two models and ranged from 0.607-0.704 (exponential model) and 0.659-0.721 (non-linear model), respectively. Mean distance travelled varied from 57-122 $\mathrm{m}$, with a maximum dispersal of $263 \mathrm{~m}$. Overall, lower infestation indexes and adult female survival were observed in the high income neighbourhood, suggesting a lower dengue transmission risk in comparison to the suburban area and the slum. Since results show that urban structure can influence mosquito biology, specific control strategies might be used in order to achieve cost-effective Ae. aegypti control.
\end{abstract}

Key words: dengue - vectorial capacity - landscape - bionomics

The reduction of dengue transmission can be attained primarily by controlling Aedes aegypti population density by maintaining it below a critical threshold in order to avoid epidemics (Focks 2003). This species, Ae. aegypti, is highly associated with human dwellings and most often breeds in man-made containers (Macielde-Freitas et al. 2007c). Theoretically, targeting the most productive containers, i.e., the ones that host high numbers of pupae might produce a focused and cost-effective control approach, which could result in reduction of adult mosquito populations (Focks 2003).

As in other metropolitan areas throughout the world, Rio de Janeiro (RJ) is a highly heterogeneous city, with slums and luxurious houses sometimes in close proximity. One can view RJ as a complex urban mosaic, which might have several implications on life history parameters of Ae. aegypti and, consequently, on dengue transmission (Luz et al. 2003, Lourenço-de-Oliveira 2008). This report aims to evaluate container productivity, adult dispersal and daily survival rates of Ae. aegypti in the high-standard and high-income neighbourhood of $\mathrm{RJ}$, named Urca, and compare results with data gathered in similar reports of slums and suburban areas.

Financial support: $\mathrm{CNPq}$

+ Corresponding author: freitas@ioc.fiocruz.br

Received 5 March 2009

Accepted 5 August 2009
Field experiments were conducted in an isolated section of Urca $\left(22^{\circ} 56^{\prime} 43^{\prime \prime} \mathrm{S} 43^{\circ} 09^{\prime} 42^{\prime \prime} \mathrm{W}\right)$ with 1,078 premises. Urca, a sparsely populated neighbourhood (297.1 inhabitants/ha), occupies an area of 13.41 ha and is located on the base of Sugar Loaf Mountain, which is one of the country's most important tourist sites. It is characterised by regular blocks with high standard dwellings, abundant vegetation coverage, a piped water supply and garbage collection. Houses are most often in the form of duplexes with at least three dorm rooms. The houses generally have large and shaded peridomestic environments, the majority containing private pools. The field site is enclosed by Guanabara Bay and Sugar Loaf Mountain, where it is isolated from its surroundings. Thus, we expected to minimise mosquito losses by emigration during field experiments. In March 2007 and March 2008, a house index (HI) of 2.67 and 0.0 , respectively, was observed in Urca neighbourhood (SMS 2008). House-to-house surveys of 534 premises (49.53\%) were conducted during March 2007 and of 507 (47.03\%) premises during March 2008 (summer). When entry was permitted by the house owner, all containers were inspected, and immature larvae were collected and brought to the laboratory for identification by following established identification keys.

Ae. aegypti used in the mark-release-recapture (MRR) experiment were derived from a laboratory colony that is constantly renewed with field-collected eggs at least twice a year. Larvae were fed with fish food $\left(\right.$ Tetramin $\left.^{\circledR}\right)$ and raised at $25 \pm 3^{\circ} \mathrm{C}$ with $60 \pm 5$ relative humidity. Adults were fed ad libitum with $10 \%$ sucrose 
solution until the day of release. MRR experiments were performed during the dry season (September 2007) when dengue cases are often reduced in RJ. During this period, a north-western wind with an average speed of $1.4 \mathrm{Km} / \mathrm{h}$ was registered during mosquito release. Prior to release, 2-3-day-old females were split into three cohorts, each one with a different colour of fluorescent dust (Day-Glo Color Corp, Cleveland) and released at a specific point within the study area. Mated and unfed Ae. aegypti females were released outdoors, less than $1 \mathrm{~h}$ after dust-marking, in reasonably equidistant points (about $150 \mathrm{~m}$ from each other).

Captures started on the following day after release and were performed daily for 10 days. Each day, 45 houses were randomly selected for aspiration (John W Hock, Gainesville, FL), which was done for 15-20 min per house, including the peridomestic area. A BGS-Trap (BioGents $\mathrm{GmbH}$, Ragensburg), which preferentially captures blood-seeking Ae. aegypti females (Macielde-Freitas et al. 2006b), was installed in five houses, remained there throughout the experiment and was monitored daily. Eventually, aspiration was performed in a house in which a BGS-Trap was installed. Finally, 15 sticky ovitraps were installed at the boundary of the field site as an attempt to capture emigrating mosquitoes and they were examined at the end of the MRR experiment. Daily captures ended when no marked females were collected over three consecutive days. The locations of the release and collection points were geo-referenced using a global positioning system (GPS; Garmin eTrex personal navigator, Garmin International, Olathe) to calculate the distance flown by Ae. aegypti females. Mosquito flight behaviour was calculated after assessing mean distance travelled (MDT), maximum distance travelled (MAX) and flight ranges (FR) of marked individuals (Morris et al. 1991). The MDT of the three cohorts released was compared by a two-sample unpaired Student's $t$-test with Welch's correction, due to the different standard deviations measured between cohorts (Zar 1999). The probability of daily survival (PDS) was estimated by fitting the exponential and nonlinear models to data using the R.2.8.0 (R Development Core Team 2008) software, despite some evidence that Ae. aegypti mortality varies with increasing age (Gillies 1961, Buonaccorsi et al. 2003, Styer et al. 2007). PDS values of the three cohorts released were compared by a two sample $t$-test (Zar 1999). From the lower and upper 95\% limits of the confidence interval of nonlinear PDS estimation, we derived the following: (i) the average life expectancy (ALE), defined as $1 /-\log _{\mathrm{e}}$ PDS (Niebylski \& Craig 1994) and (ii) the expected proportion of mosquitoes surviving long enough to transmit dengue virus, defined as $\operatorname{PDS}^{10}$ (where 10 is the duration of the extrinsic incubation period for dengue) (Salazar et al. 2007).

Container productivity, dispersal and PDS in Urca were compared to data gathered in two other dengueendemic neighbourhoods in RJ with contrasting urban characteristics: a slum (Favela do Amorim) and a suburban area (Tubiacanga) (Table I) (Maciel-de-Freitas et al. 2007a, c). MDT values of Urca, the slum area and the suburban neighbourhoods were log-transformed be- cause this variable presented non-normal distribution and they were compared by one-way ANOVA, followed by Tukey-Kramer multiple comparisons post-test. We also used a generalised estimation equation (GEEs) to evaluate the effect of the study area (categorical variable: Tubiacanga/Amorim/Urca), season (categorical variable: dry/wet) and days after release (continuous variable) on mosquito dispersal. GEEs are linear regression models with a correction of variance caused by the blocking design (cohorts), which was fitted to our data assuming an exchangeable correlation structure (Quinn \& Keough 2002). The PDS values obtained in the three settlements were contrasted by comparing the point estimates of survival rates by a two-sample unpaired $t$-test (Zar 1999). Since there were no differences within cohorts survival in suburban and slum neighbourhoods (Maciel-de-Freitas et al. 2007a), we used the mean number of dustmarked mosquitoes collected per day to create a mean cohort, which was analysed using the R.2.8.0 (R Development Core Team 2008) software.

In March 2007, 507 premises (47.03\%) and 3,495 water-holding containers were inspected. A total of 105 pupae and 2,148 larvae of Ae. aegypti were collected in 41 positive houses, resulting in an $\mathrm{HI}$ of $8.08 \%$ and a mean of 0.056 pupae per person. The most productive containers were domestic drains and discarded plastic pots, with $59.04 \%$ e $22.85 \%$ of collected pupae, respectively (Table II). In March 2008, 534 (49.53\%) premises were inspected. A total of 60 pupae and 439 larvae of $A e$. aegypti were collected in 2,848 water-holding containers, resulting in an $\mathrm{HI}$ of $6.74 \%$ and a mean pupae to person ratio of 0.032 . The most productive containers were domestic drains and plastic pots (Table II). During this survey, 14 Aedes albopictus larvae were collected.

A total of 1,750 marked Ae. aegypti females were released. Capturing efforts lasted 10 days and an overall recapture rate of $9.77 \%$ was observed, varying from $5 \%$ (Cohort 3) to 12.2\% (Cohort 1) between cohorts. Females released in cohort 1 showed lower survival than females released in cohort $3(\mathrm{t}=3.43, \mathrm{df}=12, \mathrm{p}<0.05)$. However, no significant differences were observed in PDS values between cohorts 1 and $2(t=1.63, \mathrm{df}=13, \mathrm{p}>0.05)$ and between cohorts 2 and $3(t=1.66, d f=13, p>0.05)$. ALE varied from 1.93-4.88 days, with a maximum of around $13 \%$ of Ae. aegypti females surviving long enough to transmit dengue (Table III).

The PDS of Ae. aegypti females varied significantly between neighbourhoods. The three cohorts released in the high income area (Urca) had a lower PDS in comparison to the slum (Favela do Amorim): cohort 1: $t=20.31$, $\mathrm{df}=18, \mathrm{p}<0.05$; cohort $2: \mathrm{t}=8.22, \mathrm{df}=18, \mathrm{p}<0.05$; cohort 3: $\mathrm{t}=5.83, \mathrm{df}=19, \mathrm{p}<0.05$. When compared with the suburban neighbourhood (Tubiacanga), two cohorts from Urca had lower survival rates (cohort 1: $\mathrm{t}=9.16$, $\mathrm{df}=15, \mathrm{p}<0.05$; cohort 2: $\mathrm{t}=2.87, \mathrm{df}=15, \mathrm{p}<0.05)$, and one cohort was statistically similar (cohort $3: t=0.73$, $\mathrm{df}=16, \mathrm{p}>0.05)$. PDS in the slum was higher than in the suburban neighbourhood (Maciel-de-Freitas et al. 2007a).

Ae. aegypti females had an overall displacement of $82.5 \mathrm{~m}$. Higher displacement was observed for seven females, which travelled more than $260 \mathrm{~m}$ from their re- 


\section{TABLE I}

Total number of dengue cases from 2000-2008 in the three neighborhoods in Rio de Janeiro where mark-release-recapture experiments were performed

\begin{tabular}{lcccccccccc}
\hline & 2000 & 2001 & 2002 & 2003 & 2004 & 2005 & 2006 & 2007 & 2008 & Total \\
\hline High income & 1 & 17 & 69 & 3 & 0 & 1 & 13 & 46 & 74 & 224 \\
Suburban & 25 & 71 & 755 & 9 & 1 & 3 & 24 & 42 & 607 & 1,537 \\
Slum & 12 & 216 & 907 & 14 & 4 & 7 & 27 & 47 & 861 & 2,095 \\
\hline
\end{tabular}

lease point (Table IV). Cohort 3 flew longer distances than cohort $1(\mathrm{t}=4.25, \mathrm{df}=75, \mathrm{p}<0.001)$ and cohort $2(\mathrm{t}=5.91, \mathrm{df}=88, \mathrm{p}<0.001)$. No significant differences were found between distances travelled by cohorts 1 and $2(\mathrm{t}=1.72$, $\mathrm{df}=125, \mathrm{p}>0.05)$. Sticky ovitraps installed in the boundary of field site captured three females from cohort 2 and 11 females from cohort 3. Overall, Ae. aegypti females displaced similar distances in comparison with those in the suburban area $(p>0.05)$, but they presented higher displacement than those in the slum $(\mathrm{p}<0.001)$. The multivariate linear model fitted to data points for "time after release", "area (suburban)" and "area (high income)" showed significant effects on dispersal (Table V). The MDT observed in Urca was intermediary when compared with the suburban (higher) and the slum (lower) areas. Assuming that cohort correlation was negligible $(p=0.033)$, we may use the $R^{2}$ to measure the goodness-of-fit. We found $\mathrm{R}^{2}=0.40$.

These results reinforce the notion that urban characteristics may influence mosquito biology and, probably, dengue transmission. Differences observed in mosquito parameters, such as infestation levels and PDS between areas suggest a higher probability of dengue transmission in the slum area and a lower risk in the high income area. This scenario is reinforced by taking into account the recent history of dengue notifications (2000-2008) in these three neighbourhoods (Table I). Remarkably, almost 10 times more dengue cases were registered in the slum area than in the high income area during 2000-2008.

In house-to-house surveys performed in the high income area, domestic drains and abandoned plastic pots were the most productive containers, which together accounted for up to $82 \%$ of the total pupae collected. Meanwhile, the most productive container types found in slum and suburban areas were water tanks and metal drums (Maciel-de-Freitas et al. 2007c), which was expected due to the irregularity of the water supply (Barrera et al. 1993). In field studies conducted in the United States, higher infestation, measured by HI, was observed in substandard areas as compared to that in standard areas (Tinker 1964, Von Windeguth et al. 1969). The major achievement of this report is to show that, in unplanned metropolitan areas, such as RJ and several other cities throughout the world, governments may want to adopt specific control activities regarding aspects of mosquito biology in each neighbourhood, instead of a uniform control approach for the whole city (Lourenço-de-Oliveira 2008).
The pupal surveys indicated two small-sized containers that are often susceptible to the rainfall regime, domestic drains and abandoned plastic pots, as the most productive containers. These findings are in agreement with a similar pattern observed in Trinidad (Focks \& Chadee 1997). This observation suggests that the adult mosquito population may have high fluctuations during the year, a different scenario than was observed in the slum and suburban areas, where permanent and largesized containers were more productive during dry and wet seasons (Maciel-de-Freitas et al. 2007c). In the highincome area, an educational campaign to alert the public of the importance of private pools and plastic containers (buckets and pots) may result in a decrease of $35 \%$ on the adult mosquito population. On the other hand, educational campaigns might not achieve positive results easily in the slum and suburban neighbourhoods, where households have a deficient supply of piped water and, consequently, have a habit of storing large containers of water.

The vectorial capacity formula represents the daily rate of potentially infective contacts between mosquitoes and human beings (Garret-Jones 1964). In the Ae. aegypti-dengue virus system, the daily survival rate of adult females is one of the most important parameters in dengue transmission models, since small increases in survival may exponentially increase the vectorial capacity of mosquitoes (Garret-Jones 1964, Kuno 1995, Luz et al. 2003). The survival rate of Ae. aegypti females was higher in the slum when compared to the suburban site (Maciel-de-Freitas et al. 2007a). The lower PDS observed in the high-income neighbourhood, in comparison with the slum and suburban sites, is probably the end result of several components. The most obvious aspect would be the lower human density in Urca. Maciel-de-Freitas et al. (2007b) observed a tendency towards higher Ae. aegypti survival in areas with a high human density. In more crowded areas, such as slums, mosquitoes would not have to displace far to find blood meals, which would reduce their likelihood of mortality by environmental factors, such as wind and daily variations of temperature and rainfall (Clements \& Paterson 1981). Container productivity profiles may also be important to determine adult mosquito daily survival. The higher productivity of small, non-permanent containers in Urca and of large, permanent containers in the slums and suburban areas may influence larval competition. This could possibly produce adults of different sizes and affect several aspects of mosquito biology, includ- 


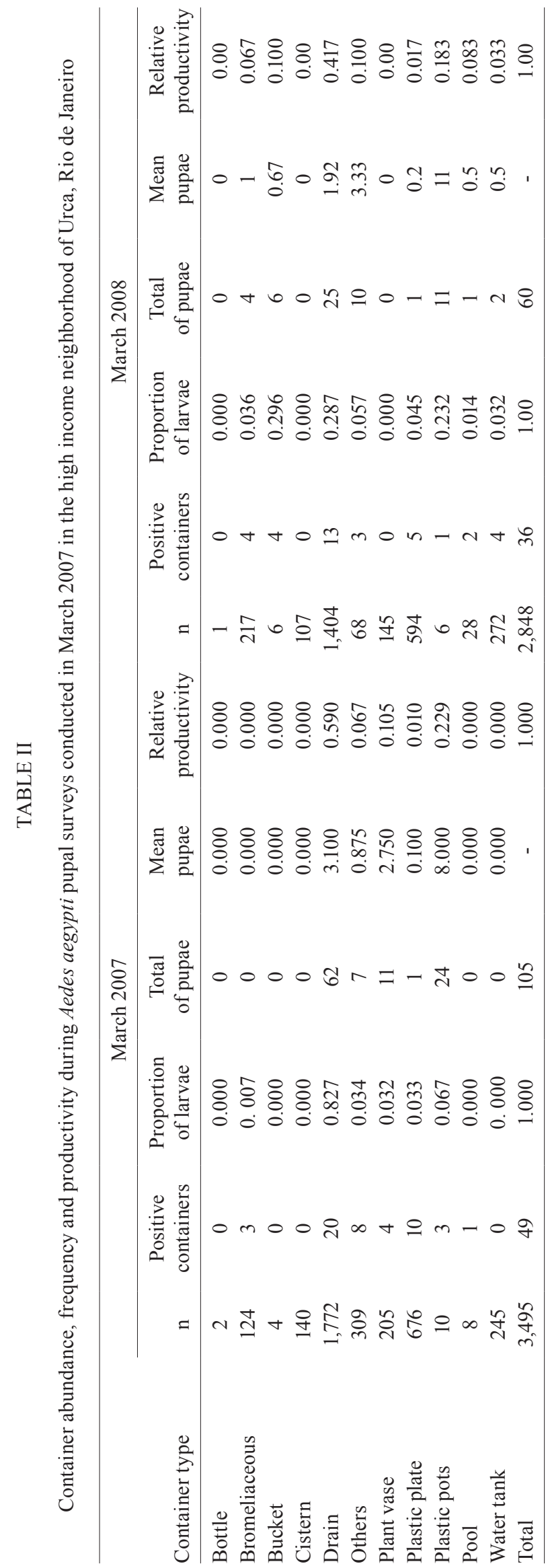

ing survival and virus susceptibility (Nasci 1986, Nasci \& Mitchell 1994, Alto et al. 2005, Maciel-de-Freitas et al. 2007b). A last explanation may depend on the habits, education and socioeconomic level of Urca households in contrast with residents of slums and suburban neighbourhoods. During pupal surveys and adult mosquito collections, health agents frequently observed commercial insecticides inside houses. Estimating the impact of this finding on mosquito survival rates seems prohibitive, but this uncontrolled variable obviously has a negative effect on Ae. aegypti longevity.

Traditionally, Ae. aegypti is regarded as a mosquito with limited FR (Harrington et al. 2005). MDT and MAX obtained in Urca reinforce this assumption and agree with previous reports (Muir \& Kay 1998, Tsuda et al. 2001, Harrington et al. 2005). Mosquitoes presented similar dispersal ratios in the high-income and suburban areas and a lower dispersal in the slum. Ae aegypti dispersal might be influenced by the availability of oviposition sites and/or blood sources (Edman et al. 1998, Maciel-de-Freitas et al. 2006a). In fact, human density in the slum (901.2 hab/ha) was higher than in the highincome (297.1 hab/ha) and suburban neighbourhoods (337.4 hab/ha). The mean number and availability of water-holding containers per house was similar among the three neighbourhoods, but the most productive containers varied within sites, especially in Urca in comparison to suburban and slum areas. The influence of the availability and spatial distribution of specific container types on mosquito dispersal is still underestimated.

Ae. aegypti dispersal has important consequences for dengue control. In Brazil, the identification of a dengue case is followed by source reduction, insecticide spraying and larvicide application in a ring area centred at the identified dengue case (Macoris et al. 2007). It is likely that Ae. aegypti control may be improved if each neighbourhood has particular radius and ring sizes in which to focus mosquito control efforts, which may be dependent of the number of oviposition sites per area and human population density. However, further investigations are needed to evaluate this hypothesis. Ae. aegypti dispersal was significantly influenced by area but not by season. The assumption that mosquito dispersal is constant over the year is surprising, since RJ experiences important seasonal trends in climate, especially in rainfall. Theoretically, the number of water-holding containers might be higher during wet seasons, resulting in lower dispersal rates (Edman et al. 1998, Lourenço-de-Oliveira et al. 2004). However, an extensive analysis of Ae. aegypti dispersal by MRR techniques also found that mosquito displacement was not influenced by seasonality (Harrington et al. 2005).

Taken together, these results strongly suggest a lower risk of dengue transmission in high-income neighbourhoods and a higher risk in slum areas. However, further investigations might address if poor neighbourhoods have higher risks of dengue transmission in metropolitan areas. Extrapolation from our conclusions must be done with caution, since we evaluated mosquito biology in only three neighbourhoods in a city with more than six million inhabitants. However, our 


\section{TABLE III}

Survival analysis for three Aedes aegypti cohorts released in Urca, Rio de Janeiro, and captured with backpack battery powered aspirators and BGS-Traps during 10 consecutive days

\begin{tabular}{lcccc}
\hline Cohort & & & & \multicolumn{2}{c}{$\begin{array}{c}\text { Alive after } 10 \text { days }^{a} \\
\%\end{array}$} \\
\hline 1 & Exponential & Buonaccorsi & ALE (Buonaccorsi) & $0.85-2.90$ \\
2 & 0.607 & $0.659(0.621-0.702)$ & $2.09-2.82$ & $0.57-5.13$ \\
3 & 0.645 & $0.677(0.597-0.743)$ & $1.93-3.36$ & $1.19-12.93$ \\
\hline
\end{tabular}

a: 10 days, dengue extrinsic incubation period; ALE: average life expectancy.

\section{TABLE IV}

Distances in meters travelled by dust-marked Aedes aegypti females captured with backpack aspirators and BGS-Trap in a high-income neighborhood of Urca, Rio de Janeiro, during September 2007

\begin{tabular}{lccc}
\hline & Cohort 1 & Cohort 2 & Cohort 3 \\
\hline Mean distance travelled & 67.48 & 57.75 & 122.31 \\
Flight range $_{50}$ & 65.6 & 46.81 & 88.45 \\
Flight range $_{90}$ & 118.7 & 81.22 & 262.3 \\
Maximum distanced travelled & 167.59 & 197.52 & 263.75 \\
\hline
\end{tabular}

\section{TABLE V}

Generalized estimating equations model for $\log$ (dispersal) of Aedes aegypti females in mark-release-recapture experiments conducted in the high income, suburban and slum districts

\begin{tabular}{lcccc}
\hline Variable & Effect & SE & Wald & $\mathrm{p}$ \\
\hline Intercept & 3.026 & 0.237 & 0.016 & $<0.001$ \\
Area (Tubiacanga $^{a}$ ) & 0.651 & 0.124 & 0.274 & $<0.001$ \\
Area $\left(\mathrm{Urca}^{b}\right.$ ) & 0.853 & 0.310 & 7.594 & $<0.001$ \\
Season (wet) & 0.018 & 0.215 & 0.007 & 0.934 \\
Time after release & 0.154 & 0.031 & 0.245 & $<0.001$ \\
Correlation $^{c}$ & 0.106 & 0.050 & 4.522 & 0.033 \\
\hline
\end{tabular}

$a$ : suburban district; $b$ : high income urban district; $c$ : correlation structure used was the "exchangeable"; SE: standard erro.

study identified that urban landscape has several implications for the life history parameters of Ae. aegypti females in the three study areas. As many other metropolitan urban areas in the tropics, RJ has unplanned urbanisation, producing an urban mosaic with slums and high-income areas in close proximity to each other. The major achievement of our report is to suggest that governments adopt specific control activities in each neighbourhood regarding aspects of mosquito biology, instead of a uniform control approach for an entire city (Lourenço-de-Oliveira 2008).

\section{ACKNOWLEDGEMENTS}

To Gabriel Sylvestre, Reginaldo Rego, Roberto Peres and Taciana Gatto, for field collections, and to Fernando Costa and Iran Nascimento, for field assistance.

\section{REFERENCES}

Alto BW, Lounibos LP, Higgs S, Juliano SA 2005. Larval competition differentially affects arbovirus infection in Aedes mosquitoes. Ecology 86: 3279-3288.

Barrera R, Avila J, Gonzales-Tellez S 1993. Unreliable supply of potable water and elevated Aedes aegypti larval indices: a causal relationship? J Am Mosq Control Assoc 9: 189-195.

Buonaccorsi JP, Harrington LC, Edman JD 2003. Estimation and comparison of mosquito survival rates with release-recaptureremoval data. J Med Entomol 40: 6-17.

Clements AN, Paterson GD 1981. The analysis of mortality and survival rates in wild populations of mosquitoes. $J$ Appl Ecol 18: 373-399.

Edman JD, Scott TW, Costero A, Morrisson AC, Harrington LC, Clark GG 1998. Aedes aegypti (Diptera: Culicidae) movement influenced by availability of oviposition sites. J Med Entomol 35: 578-583.

Focks DA 2003. A review of entomological sampling methods and indicators for dengue vectors. Special Program for Research and Training in Tropical Diseases (TDR), UNICEF, UNDP, World Bank, World Health Organization. Available from: http://www. who.int/tdr/publications/publications/pdf/dengue_vectors.pdf.

Focks DA, Chadee DD 1997. Pupal survey: an epidemiologically significant surveillance method for Aedes aegypti: an example using data from Trinidad. Am J Trop Med Hyg 56: 159-167.

Garret-Jones C 1964. The human blood index of malaria vectors in relation to epidemiological assessment. Bull World Health Organ 30: 241-261.

Gillies MT 1961. Studies on the dispersion and survival of Anopheles gambiae Giles in East Africa, by means of marking and releasing experiments. Bull Entomol Res 52: 99-127.

Harrington LC, Scott TW, Lerdthusnee K, Coleman RC, Costero A, Clark GG, Jones JJ, Kitthawee S, Kittayapong P, Sithiprasasna R, Edman JD 2005. Dispersal of the dengue vector Aedes aegypti within and between rural communities. Am J Trop Med Hyg 72: 209-220.

Kuno G 1995. Review of the factors modulating dengue transmission. Epideomol Rev 17: 321-335.

Lourenço-de-Oliveira R 2008. Rio de Janeiro against Aedes aegypti: yellow fever in 1908 and dengue in 2008. Mem Inst Oswaldo Cruz 103: 627-628. 
Lourenço-de-Oliveira R, Vazeille M, Filippis AMB, Failloux AB 2004. Aedes aegypti in Brazil: genetically differentiated populations with high susceptibility to dengue and yellow fever viruses. Trans R Soc Trop Med Hyg 98: 43-54.

Luz PM, Codeço CT, Massad E, Struchiner CJ 2003. Uncertainties regarding dengue modeling in Rio de Janeiro, Brazil. Mem Inst Oswaldo Cruz 98: 871-878.

Maciel-de-Freitas R, Brocki-Neto RW, Gonçalves JM, Codeço CT, Lourenço-de-Oliveira R 2006a. Movement of dengue vectors between human modified environment and urban forest in Rio de Janeiro. J Med Entomol 43: 1112-1120.

Maciel-de-Freitas R, Codeço CT, Lorenço-de-Oliveira R 2007a. Daily survival rates and dispersal of Aedes aegypti females in Rio de Janeiro, Brazil. Am J Trop Med Hyg 76: 659-665.

Maciel-de-Freitas R, Codeço CT, Lourenço-de-Oliveira R 2007b. Body size-associated survival and dispersal rates of Aedes aegypti in Rio de Janeiro. Med Vet Entomol 21: 284-292.

Maciel-de-Freitas R, Eiras AE, Lourenço-de-Oliveira R 2006b. Field evaluation of effectiveness of the BG-Sentinel, a new trap for capturing adult Aedes aegypti (Diptera: Culicidae). Mem Inst Oswaldo Cruz 101: 321-325.

Maciel-de-Freitas R, Marques WA, Peres RC, Cunha SP, Lourençode-Oliveira R 2007c. Variation in Aedes aegypti (Diptera: Culicidae) container productivity in a slum and a suburban district of Rio de Janeiro during dry and wet seasons. Mem Inst Oswaldo Cruz 102: 489-496.

Macoris MLG, Andrighetti MTM, Otrera VCG, Carvalho LR, Caldas Júnior AL, Brogdon WG 2007. Association of insecticide use and alteration on Aedes aegypti susceptibility status. Mem Inst Oswaldo Cruz 102: 895-900.

Morris CD, Larson VL, Lounibos LP 1991. Measuring mosquito dispersal for control programs. J Am Mosq Control Assoc 7: 608-615.

Muir LE, Kay BH 1998. Aedes aegypti survival and dispersal estimated by mark-release-recapture in northern Australia. Am J Trop Med Hyg 58: 277-282.
Nasci RS 1986. The size of emerging and host-seeking Aedes aegypti and the relation of size to blood-feeding success in the field. $J$ Am Mosq Control Assoc 2: 61-62.

Nasci RS, Mitchell CJ 1994. Larval diet, adult size and susceptibility of Aedes aegypti (Diptera: Culicidae) to infection with Ross River virus. J Med Entomol 31: 123-126.

Niebylski ML, Craig GB 1994. Dispersal and survival of Aedes albopictus at a scrap tire yard in Missouri. J Am Mosq Control Assoc 10: 339-343.

Quinn GP, Keough MJ 2002. Experimental design and data analysis for biologists, Cambridge University Press, Cambridge, 537 pp.

$\mathrm{R}$ Development Core Team 2008. $R$ 2.8.0: a language and environment for statistical computing, R Foundation for Statistical Computing, Vienna.

Salazar MI, Richardson JH, Sánchez-Vargas I, Olson KE, Beaty BJ 2007. Dengue virus type 2: replication and tropisms in orally infected Aedes aegypti mosquitoes. BMC Microbiol 7: 9.

SMS - Secretaria Municipal de Saúde do Rio de Janeiro 2008. Dados epidemiológicos. Casos de incidência de dengue por bairro e mês e por bairro e semana no Município do RJ. Available from: http://www.saude.rio.rj.gov.br/saude/pubsms/media/tab_incidengue2008.htm.

Styer LM, Carey JR, Wang JL, Scott TW 2007. Mosquitoes do senesce: departure from the paradigm of constant mortality. Am J Trop Med Hyg 76: 111-117.

Tinker ME 1964. Larval habitat of Aedes aegypti (L.) in the United States. Mosq News 24: 426-432.

Tsuda Y, Takagi M, Wang S, Wang Z, Tang L 2001. Movement of Aedes aegypti (Diptera: Culicidae) released in a small isolated village on Hainan Island China. J Med Entomol 38: 93-98.

Von Wide Guth DL, Eliason DA, Kilpatrick JW, Jakob WL 1969. The transitory nature of Aedes aegypti larval habitats in an urban situation. Mosq News 29: 495-496.

Zar JH 1999. Biostatistical analysis, 4th ed., Prentice Hall, London, $663 \mathrm{pp}$ 